\title{
On the Limiting Regularity Result of some Nonlinear Elliptic Equations
}

\author{
M. Kbiri Alaoui, D. Meskine and A. Souissi
}

Abstract. We shall prove the limiting regularity $W_{0}^{1, \frac{N(p-1)}{N-1}}(\Omega)$ of solutions of some nonlinear elliptic problems with right hand side in $\operatorname{LLog}^{\alpha} L(\Omega)$ and $\alpha \geq \frac{N-1}{N}$. Also, an improved regularity is given when $\alpha<\frac{N-1}{N}$.

Keywords. Orlicz-Sobolev spaces, truncations, regularity of solutions.

Mathematics Subject Classification (2000). Primary 49N60, secondary 74G40, 35B65.

\section{Introduction}

We deal with boundary value problems

$$
\left\{\begin{aligned}
\mathcal{A}(u):=-\operatorname{div}(a(\cdot, u, \nabla u)) & =f & & \text { in } \Omega \\
u & =0 & & \text { on } \partial \Omega,
\end{aligned}\right.
$$

where $\Omega$ is a regular bounded domain of $\mathbb{R}^{N}, N \geq 2, a: \Omega \times \mathbb{R} \times \mathbb{R}^{N} \rightarrow \mathbb{R}^{N}$ is a Carathéodory function (that is, measurable with respect to $x$ in $\Omega$ for every $(s, \xi)$ in $\mathbb{R} \times \mathbb{R}^{N}$, and continuous with respect to $(s, \xi)$ in $\mathbb{R} \times \mathbb{R}^{N}$ for almost every $x$ in $\Omega$ ). We assume that there exist a real positive constant $\nu>0$, a nonnegative function $k$ in $L^{p^{\prime}}(\Omega), p^{\prime}=\frac{p}{p-1}$, where $2-\frac{1}{N}<p \leq N$, such that for almost every $x$ in $\Omega$, for every $s$ in $\mathbb{R}$, for every $\xi$ and $\xi^{*}$ in $\mathbb{R}^{N}$ :

$$
\begin{gathered}
a(x, s, \xi) \xi \geq \nu|\xi|^{p} \\
{\left[a(x, s, \xi)-a\left(x, s, \xi^{*}\right)\right]\left[\xi-\xi^{*}\right]>0, \quad \xi \neq \xi^{*}} \\
|a(x, s, \xi)| \leq k(x)+|s|^{p-1}+|\xi|^{p-1} .
\end{gathered}
$$

The use of the $\operatorname{LLog}^{\alpha} L(\Omega)$ space to study the problem (E) in the linear case, is

M. Kbiri Alaoui: LERMA, Ecole Mohammadia d'Ingénieurs Avenue Ibn Sina B.P. 765, Agdal, Rabat, Maroc; mka_la@yahoo.fr

D. Meskine: Ecole Supérieure de Technologie Essaouira, BP 383, Essaouira, Maroc; driss.meskine@laposte.net

A. Souissi: GAN, Département de Mathématiques et d'Informatique, Faculté des Sciences, Avenue Ibn Battouta, B.P 1014 Rabat, Maroc; souissi@fsr.ac.ma 
early introduced by G. Stampacchia in [14] (for the case $\alpha=\frac{N-1}{N}$ ), by A. Passareli di Napoli and C. Sbordonne in [13] (for $0<\alpha \leq 1$ ) and recently by A. Fiorenza and M. Krebec in [7] for the case $\alpha \geq \frac{N-1}{N}$. In the nonlinear case, the particular situations were given in [4]. Another approach to reach the limiting regularity was given in [3].

Our main result consists in reaching the limiting regularity $W_{0}^{1, \bar{q}}(\Omega), \bar{q}=$ $\frac{N(p-1)}{N-1}$ with $f$ belonging to the space $\operatorname{LLog}^{\alpha} L(\Omega), \alpha \geq \frac{N-1}{N}$ in the nonlinear case.

For the sake of simplicity, we restrict our studies to the $p$-Laplacian problem model, i.e., $a(\cdot, u, \nabla u)=|\nabla u|^{p-2} \nabla u$.

\section{Preliminaries}

We list some well known results about Orlicz and Orlicz-Sobolev spaces.

2.1. Let $M: \mathbb{R}^{+} \rightarrow \mathbb{R}^{+}$be an $\mathrm{N}$-function, i.e., $M$ is continuous, convex with $M(t)>0$ for $t>0, \frac{M(t)}{t} \rightarrow 0$ as $t \rightarrow 0$ and $\frac{M(t)}{t} \rightarrow \infty$ as $t \rightarrow \infty$. Equivalently, $M$ admits the representation $M(t)=\int_{0}^{t} a(s) d s$, where $a: \mathbb{R}^{+} \rightarrow$ $\mathbb{R}^{+}$is nondecreasing, right continuous, with $a(0)=0, a(t)>0$ for $t>0$ and $a(t) \rightarrow \infty$ as $t \rightarrow \infty$. The $\mathrm{N}$-function $\bar{M}$ conjugate to $M$ is defined by $\bar{M}(t)=$ $\int_{0}^{t} \bar{a}(s) d s, \bar{a}: \mathbb{R}^{+} \rightarrow \mathbb{R}^{+}$is given by $\bar{a}(t)=\sup \{s: a(s) \leq t\}$ (see $\left.[1,10]\right)$. The $\mathrm{N}$-function is said to satisfy the $\Delta_{2}$-condition if, for some $k>0$,

$$
M(2 t) \leq k M(t) \quad \forall t \geq 0 .
$$

If (2.1) holds only for $t \geq t_{0}>0$, then $M$ is said to satisfy the $\Delta_{2}$-condition near infinity.

We will extend these N-functions into even functions on all $\mathbb{R}$.

2.2. Let $\Omega$ be an open subset of $\mathbb{R}^{N}$. The Orlicz class $K_{M}(\Omega)$ (resp. the Orlicz space $L_{M}(\Omega)$ ) is defined as the set of (equivalences classes of) real valued measurable functions $u$ on $\Omega$ such that $\int_{\Omega} M(u(x)) d x<+\infty\left(\operatorname{resp} . \int_{\Omega} M\left(\frac{u(x)}{\lambda}\right) d x<\right.$ $+\infty$ for some $\lambda>0)$. $L_{M}(\Omega)$ is a Banach space under the norm

$$
\|u\|_{M, \Omega}=\inf \left\{\lambda>0: \int_{\Omega} M\left(\frac{u(x)}{\lambda}\right) d x \leq 1\right\}
$$

and $K_{M}(\Omega)$ is a convex subset of $L_{M}(\Omega)$.

The closure in $L_{M}(\Omega)$ of the set of bounded measurable functions with compact support in $\bar{\Omega}$ is denoted by $E_{M}(\Omega)$. The equality $E_{M}(\Omega)=L_{M}(\Omega)$ holds if and only if $M$ satisfies the $\Delta_{2}$-condition, for all $t$ or for $t$ large according to whether $\Omega$ has infinite measure or not. The dual of $E_{M}(\Omega)$ can be identified with $L_{\bar{M}}(\Omega)$ by means of pairing $\int_{\Omega} u(x) v(x) d x$ and the dual norm on $L_{\bar{M}}(\Omega)$ is equivalently to $\|u\|_{\bar{M}, \Omega}$. 
The space $L_{M}(\Omega)$ is reflexive if and only if $M$ and $\bar{M}$ satisfy the $\Delta_{2^{-}}$ condition, for all $t$ or for $t$ large according to whether $\Omega$ has infinite measure or not.

2.3. We now turn to the Orlicz-Sobolev space. $W^{1} L_{M}(\Omega)$ (resp. $W^{1} E_{M}(\Omega)$ ) is the space of all functions such that $u$ and its distributional derivatives up to order 1 lie in $L_{M}(\Omega)$ (resp. $\left.E_{M}(\Omega)\right)$. It is a Banach space under the norm

$$
\|u\|_{1, M, \Omega}=\sum_{|\alpha| \leq 1}\left\|D^{\alpha} u\right\|_{M, \Omega}
$$

Thus, $W^{1} L_{M}(\Omega)$ and $W^{1} E_{M}(\Omega)$ can be identified with subspaces of the product of $\mathrm{N}+1$ copies of $L_{M}(\Omega)$. Denoting this product by $\prod L_{M}$, we will use the weak topologies $\sigma\left(\prod L_{M}, \prod E_{\bar{M}}\right)$ and $\sigma\left(\prod L_{M}, \prod L_{\bar{M}}\right)$.

The space $W_{0}^{1} E_{M}(\Omega)$ is defined as the (norm) closure of the Schwartz space $\mathcal{D}(\Omega)$ in $W^{1} E_{M}(\Omega)$ and the space $W_{0}^{1} L_{M}(\Omega)$ as the $\sigma\left(\prod L_{M}, \prod E_{\bar{M}}\right)$ closure of $\mathcal{D}(\Omega)$ in $W^{1} L_{M}(\Omega)$.

Let $W^{-1} L_{\bar{M}}(\Omega)$ (resp. $W^{-1} E_{\bar{M}}(\Omega)$ ) denote the space of distributions on $\Omega$ which can be written as sums of derivatives of order $\leq 1$ of functions in $L_{\bar{M}}(\Omega)$ (resp. $E_{\bar{M}}(\Omega)$ ). It is a Banach space under the usual quotient norm.

If the open set $\Omega$ has the segment property, then the space $\mathcal{D}(\Omega)$ is dense in $W_{0}^{1} L_{M}(\Omega)$ for the modular convergence and thus for the topology $\sigma\left(\prod L_{M}, \prod L_{\bar{M}}\right)$ (see $[8,9]$ ). Consequently, the action of a distribution in $W^{-1} L_{\bar{M}}(\Omega)$ on an element of $W_{0}^{1} L_{M}(\Omega)$ is well defined.

We denote by $\operatorname{LLog}^{\alpha} L(\Omega)$ the Orlicz space $L_{M}(\Omega)$ where $M(t) \sim t \ln ^{\alpha}(t)$ as $t \rightarrow \infty$.

The following abstract lemma will be applied in the following.

Lemma 2.1 ([2]). Let $F: \mathbb{R} \rightarrow \mathbb{R}$ be uniformly Lipschitzian with $F(0)=0$. Let $M$ be an $N$-function and let $u \in W_{0}^{1} L_{M}(\Omega)$ (resp. $W_{0}^{1} E_{M}(\Omega)$ ). Then $F(u) \in$ $W_{0}^{1} L_{M}(\Omega)$ (resp. $W_{0}^{1} E_{M}(\Omega)$ ). Moreover, if the set of discontinuity points of $F^{\prime}$ is finite, then

$$
\frac{\partial}{\partial x_{i}} F(u)= \begin{cases}F^{\prime}(u) \frac{\partial u}{\partial x_{i}} & \text { a.e. in }\{x \in \Omega: u(x) \notin D\} \\ 0 & \text { a.e. in }\{x \in \Omega: u(x) \in D\} .\end{cases}
$$

\section{Main result}

Let $M$ be an $\mathrm{N}$-function such that

$$
\text { (H) }\left\{\begin{array}{l}
K(s)=\left(M^{-1}(s)\right)^{p} \text { is convex and } \\
\int_{0} M \circ B^{-1}\left(\frac{1}{r^{1-\frac{1}{N}} \log ^{\alpha}\left(\frac{1}{r}\right)}\right) d r<+\infty, B(t)=t^{p-1} .
\end{array}\right.
$$


Theorem 3.1. Under the assumptions (1.1)-(1.3), $2-\frac{1}{N}<p \leq N$ and $f$ in $\operatorname{LLog}^{\alpha} L(\Omega)$ with $\alpha \geq \frac{N-1}{N}$, there exists at least a weak solution $u \in W_{0}^{1, \bar{q}}(\Omega)$ of problem (E) where $\bar{q}=\frac{N(p-1)}{N-1}$. Moreover, if $\alpha>\frac{N-1}{N}$, then $u \in W_{0}^{1} L_{M}(\Omega)$ for every $N$-function $M$ satisfying $(\mathrm{H})$.

Remark 3.2. The proof of the last theorem allows us to obtain an improved regularity of the solution $u$ of (E) in the Orlicz-Sobolev spaces. For example,

$$
\begin{array}{ll}
u \in W_{0}^{1} L_{M}(\Omega), M(t)=\frac{t^{\bar{q}}}{\log ^{\sigma}(e+t)}, & \text { for all } \sigma>1-\frac{\alpha N}{N-1} \text { if } \alpha \in\left[0, \frac{N-1}{N}[\right. \\
u \in W_{0}^{1} L_{M}(\Omega), M(t)=t^{\bar{q}} \log ^{\sigma}(e+t), & \text { for all } \sigma<\frac{\alpha N}{N-1}-1 \text { if } \alpha>\frac{N-1}{N} .
\end{array}
$$

For the case $\alpha=\frac{N-1}{N}, p<N$, the regularity $W_{0}^{1, \bar{q}}(\Omega)$ is optimal.

Hereafter, we denote by $\mathcal{X}_{N}$ the real number defined by $\mathcal{X}_{N}=N C_{N}^{\frac{1}{N}}, C_{N}$ is the measure of the unit ball of $\mathbb{R}^{N}, \mu(t)=$ meas $\{|u|>t\}$.

The following lemma (see [15] for the general case) plays an essential role for estimation of the approximate solutions of the problem .

Lemma 3.3. Let $u \in W_{0}^{1, p}(\Omega), 1<p<+\infty$. Then

$$
-\mu^{\prime}(t) \geq \mathcal{X}_{N} \mu(t)^{1-\frac{1}{N}}\left(-\frac{1}{\mathcal{X}_{N} \mu(t)^{1-\frac{1}{N}}} \frac{d}{d t} \int_{\{|u|>t\}}|\nabla u|^{p} d x\right)^{-\frac{1}{p-1}} .
$$

Proof of Theorem 3.1. If $\alpha>\frac{N-1}{N}, 2-\frac{1}{N}<p \leq N$, then we consider the approximate problem

$$
\left\{\begin{aligned}
\mathcal{A}\left(u_{n}\right):=-\operatorname{div}\left(a\left(\cdot, u_{n}, \nabla u_{n}\right)\right) & =f_{n} \quad \text { in } \Omega \\
u_{n} & \in W_{0}^{1, p}(\Omega),
\end{aligned}\right.
$$

where $\left(f_{n}\right)$ is a smooth sequence of functions satisfying $f_{n} \rightarrow f$ in $L_{H}(\Omega)$ for the modular convergence, $H(t)=t \log ^{\alpha}(1+t)$.

Let $\varphi$ be a truncation defined by

$$
\varphi(\xi)= \begin{cases}0, & 0 \leq \xi \leq t \\ \frac{1}{h}(\xi-t), & t<\xi<t+h \\ 1, & \xi \geq t+h \\ -\varphi(-\xi), & \xi<0\end{cases}
$$

for all $t, h>0$. Without loss of generality, we omit the index $n$. Using $v=\varphi(u)$ as a test function in (3.1), we obtain

$$
\begin{gathered}
\int_{\Omega} a(\cdot, u, \nabla u) \nabla u \varphi^{\prime}(u) d x=\int_{\Omega} f \varphi(u) d x \\
\frac{1}{h} \int_{\{t<|u|<t+h\}}|\nabla u|^{p} d x \leq C \int_{\{|u| \geq t+h\}} f d x .
\end{gathered}
$$


And letting $h \rightarrow 0$, we have

$$
-\frac{d}{d t} \int_{\{|u|>t\}}|\nabla u|^{p} d x \leq C \int_{\{|u| \geq t\}} f d x .
$$

By using Lemma 3.3 we obtain (supposing $-\mu^{\prime}(t)>0$ which does not affected the proof)

$$
\frac{1}{\mu^{\prime}(t)} \frac{d}{d t} \int_{\{|u|>t\}}|\nabla u|^{p} d x \leq\left(-\frac{1}{\mathcal{X}_{N} \mu(t)^{1-\frac{1}{N}}} \frac{d}{d t} \int_{\{|u|>t\}}|\nabla u|^{p} d x\right)^{\frac{p}{p-1}}
$$

or equivalently

$$
\left(\frac{1}{\mu^{\prime}(t)} \frac{d}{d t} \int_{\{|u|>t\}}|\nabla u|^{p} d x\right)^{\frac{1}{p}} \leq\left(-\frac{1}{\mathcal{X}_{N} \mu(t)^{1-\frac{1}{N}}} \frac{d}{d t} \int_{\{|u|>t\}}|\nabla u|^{p} d x\right)^{\frac{1}{p-1}}
$$

Let $M$ be an $\mathrm{N}$-function satisfying $(\mathrm{H})$. Jensen's inequality involves

$$
K\left(\frac{\int_{\{t<|u|<t+h\}} M(|\nabla u|)}{-\mu(t+h)+\mu(t)}\right) \leq \frac{\int_{\{t<|u|<t+h\}}(|\nabla u|)^{p}}{-\mu(t+h)+\mu(t)} .
$$

Then

$$
\begin{aligned}
M^{-1}\left(\frac{1}{\mu^{\prime}(t)} \frac{d}{d t} \int_{\{|u|>t\}} M(|\nabla u|) d x\right) & \leq\left(\frac{1}{\mu^{\prime}(t)} \frac{d}{d t} \int_{\{|u|>t\}}|\nabla u|^{p} d x\right)^{\frac{1}{p}} \\
& \leq\left(-\frac{1}{\mathcal{X}_{N} \mu(t)^{1-\frac{1}{N}}} \frac{d}{d t} \int_{\{|u|>t\}}|\nabla u|^{p} d x\right)^{\frac{1}{p-1}} .
\end{aligned}
$$

Therefore we have

$-\frac{d}{d t} \int_{\{|u|>t\}} M(|\nabla u|) d x \leq\left(-\mu^{\prime}(t)\right) M\left(\left(-\frac{1}{\mathcal{X}_{N} \mu(t)^{1-\frac{1}{N}}} \frac{d}{d t} \int_{\{|u|>t\}}|\nabla u|^{p} d x\right)^{\frac{1}{p-1}}\right)$.

Combining with (3.3) and the fact that the function $t \rightarrow \int_{\{|u|>t\}} M(|\nabla u|) d x$ is absolutely continuous, we obtain

$$
\begin{aligned}
\int_{\Omega} M(|\nabla u|) d x & =\int_{0}^{+\infty}\left(-\frac{d}{d t} \int_{\{|u|>t\}} M(|\nabla u|) d x\right) d t \\
& \leq \int_{0}^{+\infty}\left(-\mu^{\prime}(t)\right) M\left(\left(\frac{C \int_{\{|u| \geq t\}} f d x}{\mathcal{X}_{N} \mu(t)^{1-\frac{1}{N}}}\right)^{\frac{1}{p-1}}\right) d t \\
& \leq \frac{1}{C^{\prime}} \int_{0}^{C^{\prime}|\Omega|} M\left(\left(\frac{C}{r^{1-\frac{1}{N}} \log ^{\alpha}\left(\frac{1}{r}\right)}\right)^{\frac{1}{p-1}}\right) d r<\infty
\end{aligned}
$$


where $C^{\prime}=\left(\frac{\mathcal{X}_{N}}{C}\right)^{N^{\prime}}$, and the last inequality is obtained by using the Hölder inequality on $\int_{\{|u| \geq t\}} f d x$.

Finally, we deduce that $\left(\nabla u_{n}\right)_{n \geq 0}$ is bounded in $\left(L_{M}(\Omega)\right)^{N}$ for every N-function satisfying $(\mathrm{H})$. In particular, $\left(\nabla u_{n}\right)_{n \geq 0}$ is bounded in $\left(L^{\bar{q}}(\Omega)\right)^{N}$. As in [4], the almost everywhere convergence of the gradients can be obtained and the proof of theorem follows with the same way.

We deal now with the case $\alpha=\frac{N-1}{N}$ and $2-\frac{1}{N}<p<N$. We recall that the authors in [4] have proved some regularity result but by assuming that $\alpha=\frac{N}{N-1}$ and $p=N$. Consider now the following approximate problems:

$$
\left\{\begin{aligned}
-\operatorname{div}\left(a\left(x, u_{n}, \nabla u_{n}\right)\right)-\frac{1}{n} \operatorname{div}\left(\left|\nabla u_{n}\right|^{N-2} \nabla u_{n}\right) & =f_{n} \text { in } \Omega \\
u_{n} & \in W_{0}^{1, N}(\Omega) .
\end{aligned}\right.
$$

The solutions $u_{n}$ exist thanks to the Leray-Lions theorem (see [11]). Taking $v=u_{n}$ as test function in the problem (3.4), we have

$$
\int_{\Omega}\left|\nabla u_{n}\right|^{p} d x+\frac{1}{n} \int_{\Omega}\left|\nabla u_{n}\right|^{N} d x \leq 2\left\|f_{n}\right\|\left\|_{A}\right\| u_{n}\left\|_{\bar{A}} \leq C\right\| u_{n} \|_{W_{0}^{1, N}}
$$

where we have used the continuous and optimal injection $W_{0}^{1, N}(\Omega) \hookrightarrow L_{\bar{A}}(\Omega)$ with $\bar{A}(t)=e^{t^{N^{\prime}}}-1$ (see [5]). Then we deduce $\frac{1}{n}\left(\int_{\Omega}\left|\nabla u_{n}\right|^{N} d x\right)^{\frac{N-1}{N}} \leq C$. Let now $\phi \in W_{0}^{1, N}(\Omega)$ as test function, one has

$$
\int_{\Omega}\left|\nabla u_{n}\right|^{p-2} \nabla u_{n} \nabla \phi d x+\frac{1}{n} \int_{\Omega}\left|\nabla u_{n}\right|^{N-2} \nabla u_{n} \nabla \phi d x=\int_{\Omega} f_{n} \phi d x,
$$

SO

$$
\int_{\Omega}\left|\nabla u_{n}\right|^{p-2} \nabla u_{n} \nabla \phi d x \leq\left.\left|\frac{1}{n} \int_{\Omega}\right| \nabla u_{n}\right|^{N-2} \nabla u_{n} \nabla \phi d x \mid+C\|\phi\|_{W_{0}^{1, N}} \leq C\|\phi\|_{W_{0}^{1, N}}
$$

which implies, thanks to [6, Theorem 4.1], that $\int_{\Omega}\left|\nabla u_{n}\right|^{\bar{q}} d x \leq C$, where here and below $C$ denote positive constants not depending on $n$. Therefore, we can see that there exist a measurable function $u \in W_{0}^{1, \bar{q}}(\Omega)$ and a subsequence also denoted $\left(u_{n}\right)_{n}$,

$$
\begin{aligned}
u_{n} & \rightarrow u & & \text { weakly in } W_{0}^{1, \bar{q}}(\Omega) \\
T_{k}\left(u_{n}\right) & \rightarrow T_{k}(u) & & \text { weakly in } W_{0}^{1, p}(\Omega),
\end{aligned}
$$

where $T_{k}$ is the usual truncation defined by $T_{k}(s)=\max (-k, \min (k, s))$, for all $s \in \mathbb{R}$, for all $k \geq 0$. Let $v \in \mathcal{D}(\Omega)$ and choose the test function $T_{k}\left(u_{n}-v\right), n>$ $k+\|v\|_{\infty}$, in the approximate problem, we have

$$
\begin{aligned}
& \int_{\Omega}\left|\nabla u_{n}\right|^{p-2} \nabla u_{n} \nabla T_{k}\left(u_{n}-v\right) d x+\frac{1}{n} \int_{\Omega}\left|\nabla u_{n}\right|^{N-2} \nabla u_{n} \nabla T_{k}\left(u_{n}-v\right) d x \\
= & \int_{\Omega} f_{n} T_{k}\left(u_{n}-v\right) d x
\end{aligned}
$$


which we rewrite as follows:

$$
\begin{aligned}
& \int_{\Omega}\left(\left|\nabla u_{n}\right|^{p-2} \nabla u_{n}-|\nabla v|^{p-2} \nabla v\right) \nabla T_{k}\left(u_{n}-v\right) d x \\
& +\int_{\Omega}|\nabla v|^{p-2} \nabla v \nabla T_{k}\left(u_{n}-v\right) d x \\
& +\frac{1}{n} \int_{\Omega}\left(\left|\nabla u_{n}\right|^{N-2} \nabla u_{n}-|\nabla v|^{N-2} \nabla v\right) \nabla T_{k}\left(u_{n}-v\right) d x \\
& +\frac{1}{n} \int_{\Omega}|\nabla v|^{N-2} \nabla v \nabla T_{k}\left(u_{n}-v\right) d x \\
& =\int_{\Omega} f_{n} T_{k}\left(u_{n}-v\right) d x .
\end{aligned}
$$

This obviously gives

$\int_{\Omega}|\nabla v|^{p-2} \nabla v \nabla T_{k}\left(u_{n}-v\right) d x+\frac{1}{n} \int_{\Omega}|\nabla v|^{N-2} \nabla v \nabla T_{k}\left(u_{n}-v\right) d x \leq \int_{\Omega} f_{n} T_{k}\left(u_{n}-v\right) d x$.

By using the fact that $T_{k}\left(u_{n}-v\right) \rightarrow T_{k}(u-v)$ weakly in $W_{0}^{1, p}(\Omega)$, we obtain

$$
\int_{\Omega}|\nabla v|^{p-2} \nabla v \nabla T_{k}(u-v) d x \leq \int_{\Omega} f T_{k}(u-v) d x, \quad \forall v \in \mathcal{D}(\Omega),
$$

By the density argument the last inequality remains true for all $v \in W_{0}^{1, p}(\Omega) \cap$ $L^{\infty}(\Omega)$.

To prove that $u$ is a weak solution of the problem (E), we follow the technique used in [12]. Let $h$ and $k$ be positive real numbers, let $t$ belong to $(-1,1)$ and let $\psi$ be a function in $W_{0}^{1, p}(\Omega) \cap L^{\infty}(\Omega)$. Choose $\phi=T_{h}(u)+t T_{k}(u-\psi)$ in the previous inequality, we have $u$ is a so-called entropy solution of (E) which completes the proof of the theorem.

\section{References}

[1] Adams, R., Sobolev spaces. New York: Academic Press 1975.

[2] Benkirane, A. and Elmahi, A., Almost everywhere convergence of the gradients of solutions to elliptic equations in Orlicz spaces and application. Nonlin. Anal. 28 (1997)(11), $1769-1784$.

[3] Benkirane, A. and Kbiri Alaoui, M., Sur certaines équations elliptiques non linéaires à second membre mesure. Forum Math. 12 (2000)(4), 385 - 395.

[4] Boccardo, L. and Gallouët, T., Nonlinear elliptic equations with right-hand side measures. Comm. Partial Diff. Eqs. 17 (1992)(3,4), 641 - 655.

[5] Cianchi, A., Optimal Orlicz-Sobolev embedding. Rev. Mat. Iberoamericana 20 (2004)(2), $427-474$. 
[6] de Figueiredo, D. G., do Ó, J. M. and Ruf, B., An Orlicz space approach to superlinear elliptic systems. J. Funct. Anal. 224 (2005), 471 - 496.

[7] Fiorenza, A. and Krbec, M., Fiorenza, A. On an optimal decomposition in Zygmund spaces. Georgian Math. J. 9 (2002)(2), $271-286$.

[8] Gossez, J.-P., Some approximation properties in Orlicz-Sobolev spaces. Studia Math. 74 (1982), 17 - 24.

[9] Gossez, J.-P., Nonlinear elliptic boundary value problems for equations with rapidly (or slowly) increasing coefficients. Trans. Amer. Math. Soc. 190 (1974), $163-205$.

[10] Krasnoselśkiŭ, M. A. and Rutickiǔ, Ja. B., Convex Functions and Orlicz Spaces. Groningen: P. Noordhoff 1969.

[11] Leray, J. and Lions, J.-L., Quelques résultats de Višik sur les problèmes elliptiques nonlinéaires par les méthodes de Minty-Browder. Bull. Soc. Math. France 93 (1965), 97 - 107.

[12] Minty, G. J., On a monotonicity method for the solution of non-linear equations in Banach spaces. Proc. Nat. Acad. Sci. U.S.A. 50 (1963), $1038-1041$.

[13] Passarelli di Napoli, A. and Sbordone, C., Elliptic equations with right hand side in LLog ${ }^{\alpha}$ L. Rend. Accad. Sci. Fis. Mat. Napoli 62 (1995), $301-314$.

[14] Stampacchia, G., Some limit cases of $L^{p}$ estimates for solutions of second order elliptic equations. Comm Pure Appl. Math. 16 (1963), 505 - 510.

[15] Talenti, G., Nonlinear elliptic equations, rearrangements of functions and Orlicz spaces. Ann. Mat. Pura Appl. 120 (1979)(4), 159 - 184.

Received November 24, 2005; revised March 9, 2007 